Supporting Information

\title{
Prominence of Terahertz Acoustic Surface Plasmon excitation in Gas-Surface interaction with Metals
}

G. Bracco, L. Vattuone, M. Smerieri, G. Carraro, L. Savio, G. Paolini, G. Benedek, P. M. Echenique, M. Rocca

Correspondence to: $\underline{\text { Rocca@fisica.unige.it, }}$

This PDF file includes:

Materials and Methods

Supplementary Text with additional data

Figs. S1 to S4 with captions 


\section{Materials and Methods}

$\underline{\text { Experimental apparatus }}$

The experimental apparatus is the atom scattering set-up described in ref. ${ }^{27}$ of the main paper based on a fixed angle design between source and detector of $110^{\circ}$. The Neon beam impinges at angle $\theta_{i}$ and the scattered atoms are detected at $\theta_{f}=110^{\circ}-\theta_{i}$ within an angular acceptance of $\Delta \theta_{f}=$ $0.1^{\circ}$. The specular reflection corresponds thus to $\theta_{I}=\theta_{f}=55^{\circ}$. The scattering plane was aligned with the $(\bar{\Gamma} \bar{M})$ direction, corresponding to $\langle\overline{2} 11\rangle$ in direct space, as determined by visual inspection of the Low Energy Electron Diffraction pattern and more accurately by $\mathrm{He}$ atom scattering.

The nozzle source was kept at a pressure of 55 bar and at a temperature of $315 \mathrm{~K}$, slightly higher than room temperature, to attain a better stability. The employed gas was a mixture of $7 \%$ of $\mathrm{Ne}$ seeded in $\mathrm{He}$. In this condition, the measured incident wavevector for Ne seeded in He was 50.6 $\AA^{-1}$ for a kinetic energy $E_{i} \sim 265 \mathrm{meV}$. The electronics of the detector stage, described in ref. ${ }^{27}$ of main paper, was modified to extend the mass range of the homemade quadrupole mass filter up to 22 atomic mass units allowing thus to work in a stable way at the ${ }^{20} \mathrm{Ne}$ mass. The spectra are averaged over 1 to 5 million acquisitions to reach an acceptable signal to noise level. The position of the maximum in the inelastic spectra is convoluted over an integration window due to the angular acceptance of the apparatus, which translates into $\sim 0.2 \AA^{-1}$ in reciprocal space. The position of the maximum of the loss can, however, be determined with a much better accuracy.

To the best of our knowledge there are no literature data about the energy spread for a He/Ne mixture for pressure and temperature close to those of the present experiment. For pure beams the expected Full Width at Half Maximum (FWHM) for Ne at our value of pressure times nozzle diameter is $\sim 4 \mathrm{meV}$, i.e. about $6 \%$ of the kinetic energy of $67 \mathrm{meV}$ (ref. ${ }^{26}$ of main paper) Assuming the same relative energy resolution we would obtain for our mixture a FWHM of 16 meV. For a gas mixture the resolution should, however, be better because of the collisions between carrier and seed gases. Alternatively, we can estimate the FWHM by fitting the measured spectra with the energy width of the beam as free parameter. In this way we obtain a FWHM of $21.7 \mathrm{meV}$ using all the data points and $16 \mathrm{meV}$ when excluding the points with the worst signal to noise ratio. We can thus reasonably consider $16 \mathrm{meV}$ as an upper limit for the FWHM in our experimental conditions. 
The sample was initially cleaned with cycles of sputtering with $A r^{+}$ions of $3 \mathrm{keV}$ energy followed by annealing up to $820 \mathrm{~K}$ until a stable intensity of the $H e$ specular peak was obtained. $H e$ diffraction was exploited to accurately align the scattering plane along the crystallographic $\langle\overline{2} 11\rangle$ $(\bar{\Gamma} \bar{M})$ azimuthal direction for which the data were collected with the sample cooled to $120 \mathrm{~K}$ by filling the cryostat of the sample manipulator with liquid nitrogen. Although the base pressure was better than $10^{-10}$ mbar, some reduction of the intensity was observed with time due to adsorption of residual gases on the cold sample surface. This contamination is most probably due to physisorption of water since the initial intensity could be recovered by flashing the sample above $280 \mathrm{~K}$. Therefore, to reduce sample contamination, a protocol was adopted in which the maximum measuring times for an experimental run was limited to 6 hours.

The time-of-flight (TOF) spectra were acquired with a channel width of $250 \mathrm{~ns}$ and converted into energy or momentum taking into account the Jacobian of the transformation with the same numerical procedure adopted in the past to study surface phonons with inelastic $\mathrm{He}$ scattering (ref. 27 of main paper) and adapted for the $\mathrm{Ne}$ case.

\section{Choice of the impact energy of the Neon atoms}

The kinetic energy value of $265 \mathrm{meV}$ was chosen as the best compromise between the necessity of having enough energy to excite the ASP above the bulk phonon band, the worsening of the signal to noise ratio when reducing the Ne concentration (lower concentrations allow for higher Ne speeds), and the worsening of the energy resolution of the beam for higher nozzle temperatures.

\section{$\underline{\text { Analysis of the data }}$}

Two TOF spectra are shown in Fig. S1. They were recorded, respectively, close to the specular angle and far off-specular (left panels) and their conversion into kinetic energy difference $E_{f}-E_{i}$ with $\mathrm{E}_{\mathrm{f}}$ energy of the scattered $\mathrm{Ne}$ atoms (middle panels) and transferred wavevector parallel to the surface, $\Delta \mathrm{k}_{\|}=k\left\|_{\mathrm{f}}-k\right\|_{\mathrm{i}}$ (right panels). 

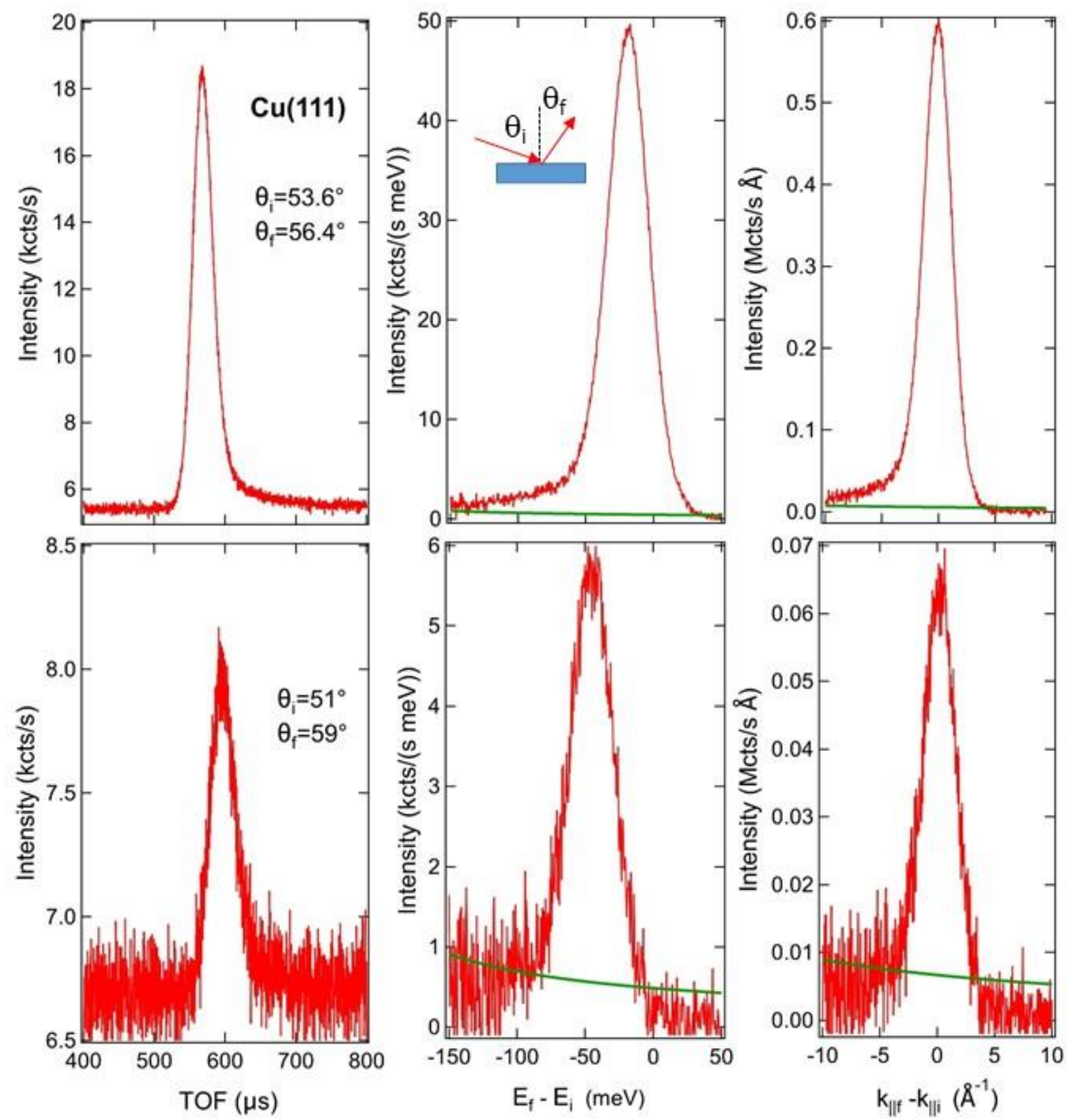

Fig. S1 Time of flight (TOF), energy and momentum spectra for $\mathrm{Ne} / \mathrm{Cu}(111)$. Upper panels incident angle $\theta_{\mathrm{i}}=53.6^{\circ}$ and scattered angle $\theta_{\mathrm{f}}=56.4^{\circ}$, lower panels for $\theta_{\mathrm{i}}=51.0^{\circ}$ and $\theta_{\mathrm{f}}=59.0^{\circ}$. Left: Scattered intensity vs. TOF. Centre: Conversion of TOF intensity after background subtraction into kinetic energy difference. Right: Same into wavevector transfer parallel to the surface. The green line indicates the limit of statistical relevance, see SM. The scattered intensity decreases with off-specular angle, see inset for the scattering geometry. 
The TOF reported in the figures has been corrected for to the delays caused by the detector crossing time and the dephasing associated with the chopper. The acquired signal in the bins of a TOF spectrum contains counts related to atoms that have been scattered off the sample surface as well as counts due to the constant background gas in the detector. Before performing the conversion of the TOF spectrum to energy or momentum exchange, the background counts B are subtracted from the TOF spectrum. This constant B value estimated from TOF is also converted and the square root value for each bin provides an estimate of the background fluctuations $F_{B}$ shown by the green curves of Fig. 1 for a one sigma relevance of the scattered signal.

The scattered intensity is acquired with a quadrupole mass spectrometer. The atoms are thereby ionized with a probability that depends on the crossing time of the ionization region and therefore proportional to the reciprocal of the atom final speed $v_{f}$ in the detector. In order to take this effect into account, in the fitting procedure all functions are multiplied by the dimensionless factor $\frac{v_{i}}{\bar{v}_{f}}$ where $v_{i}$ and $\bar{v}_{f}$ are the speed of atoms undergoing an elastic reflection and the average speed calculated for each bin of the converted spectrum, respectively.

The final and initial wave-vectors are $\boldsymbol{k}_{\mathrm{i}}$ and $\boldsymbol{k}_{\mathrm{f}}$ with parallel components given by $\boldsymbol{k}_{\| \mathrm{i}}=\boldsymbol{k}_{\mathrm{i}} \sin \theta_{i}$ and $\boldsymbol{k}_{\| \mathrm{f}}=\boldsymbol{k}_{\mathrm{f}} \sin \theta_{f}$ respectively. Moreover, initial and final energies of the atom of mass $m$ are $E_{i}=1 / 2 m v_{i}^{2}=\left(\hbar \boldsymbol{k}_{i}\right)^{2} /(2 m)$ and $E_{f}=1 / 2 m v_{f}^{2}=\left(\hbar \boldsymbol{k}_{f}\right)^{2} /(2 m)$.

From the conservation of energy and momentum parallel to the surface, the energy difference $\Delta E\left(\boldsymbol{k}_{\|}\right)$and the parallel wave-vector transfer $\left(\Delta \boldsymbol{k}_{\|}\right)$of the loss peaks are determined by:

$\Delta E\left(\boldsymbol{k}_{\|}\right)=E_{f}-E_{i}$ $\Delta \boldsymbol{k}_{\|}=\boldsymbol{k}_{f}\left\|-\boldsymbol{k}_{i}\right\|$

The above equations determine the scan curves in reciprocal space (coloured curves in Figs.1).

\section{Fitting procedure}

The ASP contribution is described by the vibronic structure of Eq. (3) if its energy falls outside of the phonon band. The intersections of the scan curve with the forward and backward branches of the ASP occur always close to $\bar{\Gamma}\left(\Delta \boldsymbol{k}_{\|}=0\right)$ and cannot be resolved. We, therefore, considered two 
vibronic structures centred at $\mathrm{E}_{\mathrm{ASP} 1}$ and $\mathrm{E}_{\mathrm{ASP} 2}$ with intensities scaled with the corresponding Bose factors, $n\left(E_{A S P 1}\right)$ and $n\left(E_{A S P 2}\right)$. In this way their intensities make up one single fitting parameter. Another parameter is the Huang-Rhys factor $S_{H R}$. The phonon energy is fixed at $\hbar \omega_{I}=21 \mathrm{meV}$, corresponding to the value with the largest coupling with the electronic degrees of freedom as observed with Inelastic Electron Tunnelling Spectroscopy performed with Scanning Tunneling Microscopy (ref. ${ }^{38}$ of main paper). The effective full width of the phonon band is taken $34 \mathrm{meV}$ since the $2 \sigma_{1}$ full width of the phonon band is convoluted with the energy spread of the Ne beam. The fits were performed for those spectra for which the ASP structures are located outside of the phonon band to avoid their possible mixing with phonons and, in turn, possible shifts in their positions due to hybridization. The phonon contribution to the inelastic intensity comes from the second Brillouin zone and is always much fainter than the one of the ASP.

Each spectrum was then fitted with a MATLAB routine to determine the value of $S_{H R}$ which minimizes the root mean square error (RMSE). The collection of the values obtained for the angles between $\theta_{\mathrm{i}}=49.6^{\circ}$ and $\theta_{\mathrm{i}}=52.5^{\circ}$ is reported in Fig. S2. The average value of $S_{H R}$ reads 0.33 .

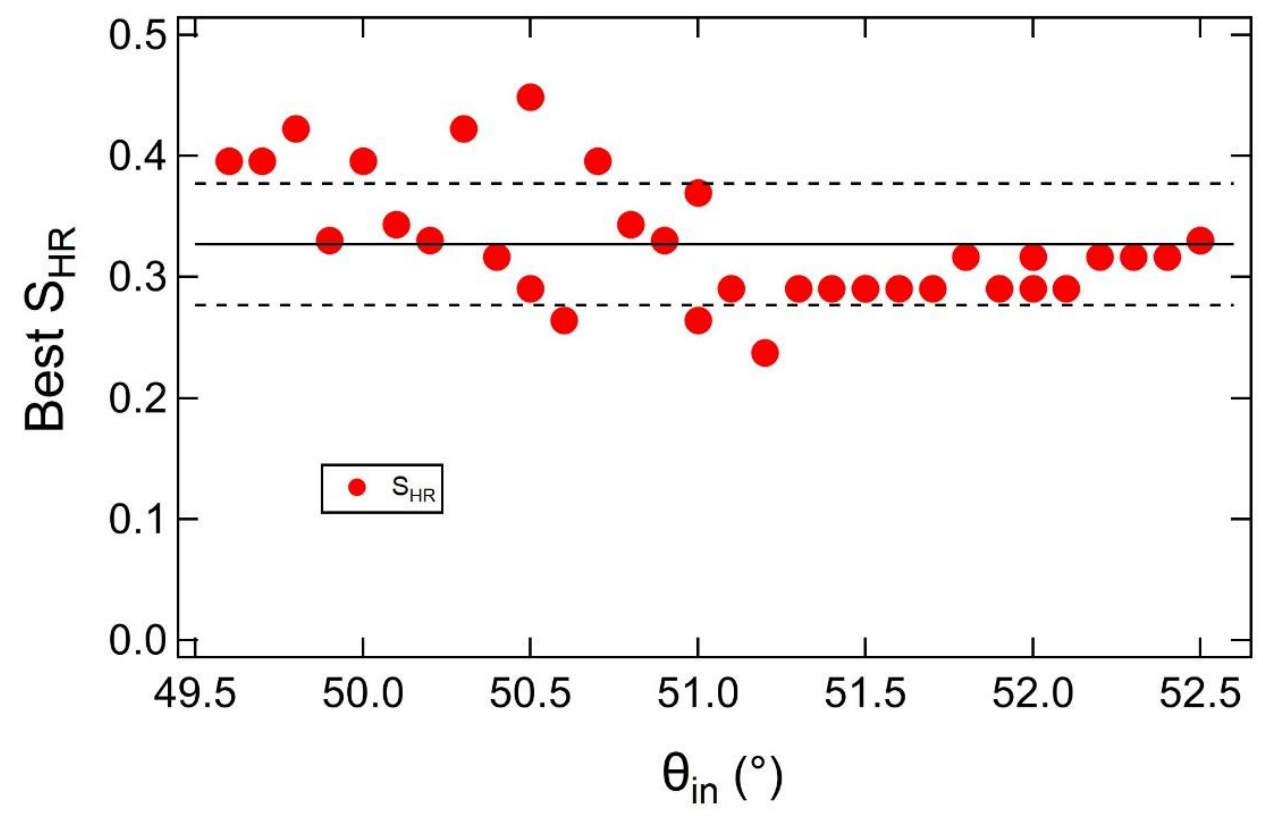

Fig. S2 $S_{H R}$ values obtained from the fit of the spectra (red dots) for which the vibronic structure falls outside of the phonon band. The solid line gives the average value while the dashed lines indicate the standard deviation. 


\section{Supplementary Text}

$\underline{\text { Additional data }}$

All experimentally recorded spectra are shown in Figs. S3 and S4. Far off-specular data are shown separately for the sake of clarity since they have a lower intensity.
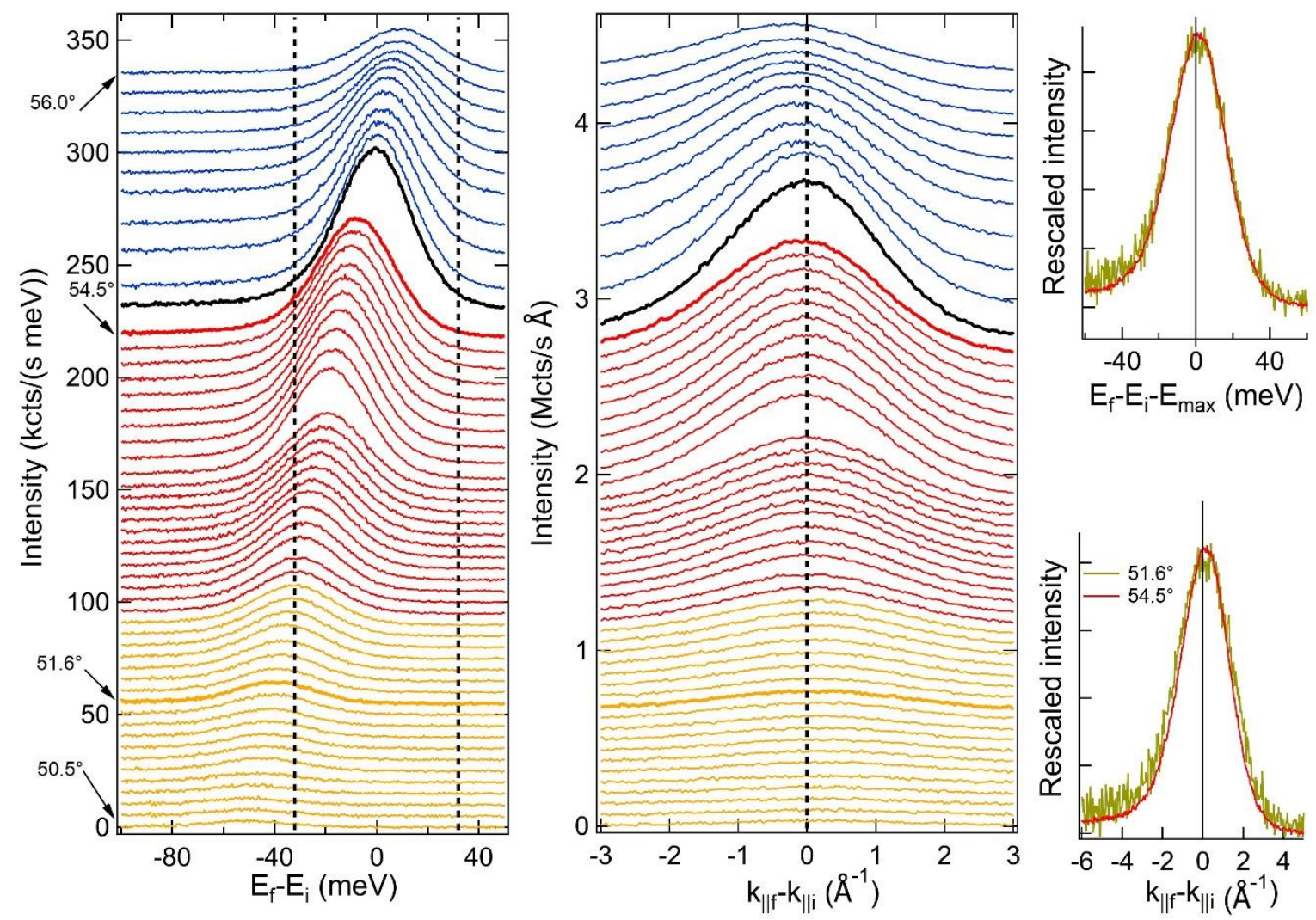

Fig. S3 Collection of experimental data recorded at $\mathrm{E}_{\mathrm{Ne}}=265 \mathrm{meV}$. Left panel: Spectra collected by rotating the crystal by $0.1^{\circ}$. $\theta_{\mathrm{i}}$ is mentioned for some spectra. Uppermost (blue) spectra correspond to $\theta_{\mathrm{i}}>\theta_{\mathrm{f}}(\Delta k=0$ on energy gain side $), 55.1^{\circ}<\theta_{\mathrm{i}}<56.0^{\circ}$. Black: specular scattering $\theta_{\mathrm{i}}=\theta_{\mathrm{f}}$ $=55.0^{\circ}$. Red and gold $\theta_{\mathrm{i}}<\theta_{\mathrm{f}}$, maxima correspond to loss energies within (red) or beyond (gold) the bulk band, bottom $50.5^{\circ}<\theta_{\mathrm{i}}<54.5^{\circ}$. The vertical dotted lines in the left panel indicate the edges of the bulk phonon bands. Central panel: Same spectra as for the left panel are reported vs $\Delta k_{\|}$. The dashed line indicates the position of $\Delta k=0$. Right panel: Thick spectra in left and central panels are rescaled and shifted for comparison to highlight the nearly constant line width with respect to energy and momentum exchange. Intensities do not show a smooth trend because we refrained from rescaling data from different runs. 

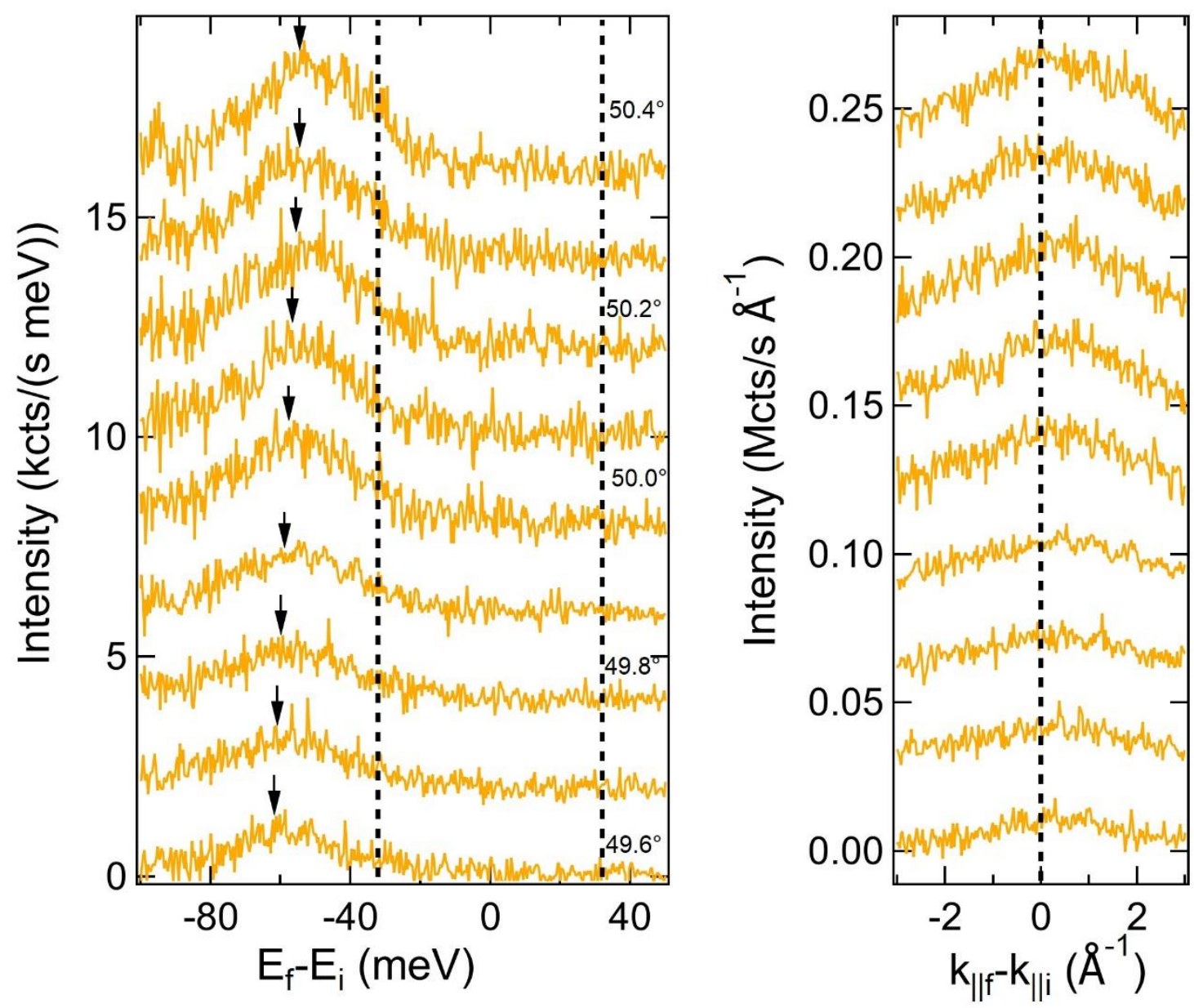

Fig. S4 Additional spectra Additional far off-specular data recorded at $\mathrm{E}_{\mathrm{Ne}}=265 \mathrm{meV}$, not reported in Fig. S3 are shown vs energy (left panel) and vs momentum (right panel). Left panel: spectra collected vs energy exchange. Right panel: Same spectra vs $\Delta k_{\|}$. In the left panel the dashes indicate the bulk phonon edges while the arrows mark the position of $\Delta k_{\|}=0$. The numbers indicate the incident angle for every second spectrum. The dashed line in the right panel is at $\Delta k_{\|}=0$.

\section{Bibliography}

(26) Toennies, J.P.; Winkelmann, K. Theoretical study of highly expanded free jets: influence of quantum effects and realistic intermolecular potential. J. Chem. Phys. 1977, 66, 3965.

(27) Pedemonte, L.; Gussoni, A.; Tatarek, R.; Bracco, G. High-resolution scattering apparatus for surface studies. Review of Scientific Instruments, 2002, 73, 4257-4263

(38) H. Gawronski, M. Mehlhorn, K. Morgenstern, Imaging phonon excitation with atomic resolution. Science 2008, 319, 930-933. 